\title{
A Practical and Secure Fault-Tolerant Conference-Key Agreement Protocol ${ }^{\star}$
}

\author{
Wen-Guey Tzeng \\ Department of Computer and Information Science \\ National Chiao Tung University \\ Hsinchu, Taiwan 30050 \\ tzeng@cis.nctu.edu.tw
}

\begin{abstract}
When a group of people wants to communicate securely over an open network, they run a conference-key protocol to establish a common conference key $K$ such that all their communications thereafter are encrypted with the key $K$. In this paper we propose a practical and provably secure fault-tolerant conference-key agreement protocol under the authenticated broadcast channel model. The adversary that attacks our protocol can be either active or passive. An active adversary (malicious participant) tries to disrupt establishment of a common conference key among the honest participants, while a passive adversary tries to learn the conference key by listening to the communication of participants. We show that a passive adversary gets no information (zero knowledge) about the conference key established by the honest participants under the assumption of a variant Diffie-Hellman decision problem. We also show that the honest participants can agree on a common conference key no matter how many participants are malicious.
\end{abstract}

\section{Introduction}

When a group of people wants to communicate securely over an open network, they run a conference-key protocol to establish a common conference key $K$ such that all their communications thereafter are encrypted with the key $K$. The first type of conference-key protocols, called conference-key distribution, is that a chairman selects a conference key and distributes the key to the participants. The second type of conference-key protocols, called conference-key agreement, is that all participants together compute a common key without a chairman. The later one is suitable for distributed environments. Conference-key protocols are also designed for various types of network connection, such as the ring connection, the star connection, the broadcast connection, etc. The conference keys of a conference-key protocol are either pre-distributed or dynamic. The conference key is fixed for a particular group of participants in a pre-distributed conferencekey protocol, while it is different for each session in a dynamic conference-key protocol. The pre-distributed conference-key protocol lacks of flexibility often.

\footnotetext{
* Research supported in part by the National Science Council, Taiwan, ROC, grant
} NSC-88-2213-E-009-053 
In this paper we propose a practical and provably secure fault-tolerant conference-key agreement protocol under the authenticated broadcast channel model. The adversary that attacks our protocol can be either active or passive. An active adversary (malicious participant) tries to disrupt establishment of a common conference key among the honest participants, while a passive adversary tries to learn the conference key by listening to the communication of participants. We tolerate the case that a malicious participant gets the conference key since the malicious participant can simply behave properly to get the key. We show that a passive adversary gets no information (zero knowledge) about the common conference key established by the honest participants under the assumption of the Diffie-Hellman decision problem. We also show that the honest participants can agree on a common conference key no matter how many participants are malicious.

We can relax the requirement of the broadcast channel being authenticated. For an un-authenticated broadcast channel, the attack from impersonators who try to impersonate participants is possible. We shall argue that our protocol is secure in this sense. We can actually use a more sophisticated commitment scheme in the protocol to achieve rigid security against impersonators. Nevertheless, in order to keep the protocol's structure and simplicity for practicality, we design our protocol as it is now.

Computing a conference key is a special case of secure multiparty computation in which a group of people evaluate a function $f\left(k_{1}, k_{2}, \cdots\right)$ securely with each person possessing a private input $k_{i}$. Therefore, it is possible to have a secure conference-key agreement protocol by the generic construction for secure multiparty computation. However, there are some distinct features in the conference-key agreement protocol. First, there are no private channels between participants, which is a general assumption in secure multiparty computation. Second, a cheater's goal in a conference-key agreement protocol is to disrupt conference-key establishment among the honest participants. This is quite different from the goal of cheaters in secure multiparty computation. Third, in multiparty computation when a cheater is found, the cheater's secret $x_{i}$, which is shared into others, is recovered by honest participants so that evaluation can proceed. In conference-key agreement, since a cheater's secret is not a necessity in computing a conference key, the cheater is simply excluded from participating when found.

There have been intensive research on conference-key protocols. For example, conference-key distribution protocols (with a chairman) have been studied in $[3,9,10,16]$, pre-distributed conference-key protocols have been studied in $[4,5,19]$, and conference-key agreement protocols have been studied in $[14,16,17,28,29]$. Most proposed protocols focus on security and message efficiency for various types of network connection. Nevertheless, they do not have the capability of fault-tolerance so that a malicious participant can easily mislead other participants to compute different keys. On the other hand, Klein et. al. [15] proposed a fault-tolerant conference-key agreement protocol. However, the protocol is quite inefficient and its security is not rigidly proved. Burmester 
and Desmedt [7] proposed an efficient (two-round) protocol (Protocol 3) for the broadcast channel with $f\left(k_{1}, k_{2}, \ldots, k_{n}\right)=g^{k_{1} k_{2}+k_{2} k_{3}+\cdots+k_{n} k_{1}} \bmod p$. They showed that an eavesdropper cannot compute the common conference key if the Diffie-Hellman decision problem is intractable. In contrast, an eavesdropper gets zero knowledge about the common conference key in our protocol if a variant Diffie-Hellman decision problem is hard. Their basic protocol cannot withstand the attack of malicious participants. In the modified Protocol 7 (authenticated key distribution), they used an interactive proof for authenticating sent messages and showed that the protocol is secure against impersonators. The number of rounds in the protocol is proportional to the number of participants, which is not practical in some cases.

\section{Model}

A user in the system is a probabilistic polynomial-time Turing machine. Each user $U_{i}$ has a secret information $x_{i}$ and a corresponding public information $y_{i}$. The system has a public directory that records the system's public parameters and each user's public information that can be accessed by every one. All users are connected by an authenticated broadcast network such that the messages sent on the network can be identified and cannot be altered, blocked or delayed. Therefore, every one can send and receive the message on the network without interruption. No private channel exists between users. A group of users who wants to establish a conference key is called the set of participants. A participant may be malicious in any way.

There are two types of adversaries. A passive adversary who is not a participant listens to the broadcast channel and tries to learn the conference key established by the honest participants. An active adversary who is a participant tries to disrupt establishment of a common conference key among the honest participants. An active adversary mainly sends "malicious" messages into the broadcast channel to fool an honest participant to believe that he has computed the same conference key as that of other honest participants, while he does not indeed. We don't care about the possibility that two or more cheating participants collaborate and result in one of them or other malicious participants not being able to compute the key. This includes the following case. A malicious participant $U_{i}$ sends "malicious" messages, but all honest participants compute the same key. Another malicious participant $U_{j}$, though receiving an incorrect key, still claims that he has had received the correct key. We tolerate this case since this type of collaboration between $U_{i}$ and $U_{j}$ do no harm to the honest participants.

A conference-key agreement protocol is secure if it can withstand attacks of passive and active adversaries. For security against a passive adversary, we mean that the adversary alone can construct a view that is computationally indistinguishable from the real conversation occurred among the participants. For security against an active adversary, we mean that if the adversary does not 
follow the protocol in any way, the probability that he can disrupt establishment of a common conference key among honest participants is negligible.

\section{Design Principles}

Our protocol is component-based, that is, our protocol uses cryptographic modules as building blocks. Component-based design has many merits. First, because of using modular design, it is easy to upgrade the components of the protocol in case that better components, in either efficiency, cost, or security, are available. Also, in case that security flaws are found in a component, we can replace the component only and need not abandon the whole established system. Second, it is easier to apply strong security analysis on the protocol. Since each component has a single security goal, we can analyze each component in the focused security features. Third, it is flexible and suitable for use in a large system. A conference may be called among the participants all over the world. Flexibility of component-based design allows each user choose adequate components for each conference session. Therefore, component-based design is suitable for large and heterogeneous systems.

Suppose that each participant $U_{i}$ holds a secret $x_{i}, 1 \leq i \leq n$. They need evaluate a function $f$ to get the conference key $K=f\left(k_{1}, k_{2}, \ldots, k_{n}\right)$, where $k_{i}$ is a randomly selected secret (sub-key) of $U_{i}$ for the conference session. In our protocol, we let each participant $U_{i}$ handle a function $f_{i}$ and the conference-key function is

$$
f\left(k_{1}, k_{2}, \ldots, k_{n}\right)=\Sigma f_{i}\left(k_{1}, k_{2}, \ldots, k_{n}\right)
$$

where $f_{i}\left(k_{1}, k_{2}, \ldots, k_{n}\right)=k_{i}$. Since the result $k_{i}$ of $f_{i}$ is independent of other parameters $k_{j}, j \neq i$, participant $U_{i}$ can broadcast messages so that other participants can evaluate $f_{i}$ in a secure multiparty computation way. As mentioned previously, our protocol is component-based. It contains the following components:

1. Component of secure multiparty computation for $f_{i}$ :

2. Component of $k_{i}$ commitment and verification:

Our conference key agreement protocol has the following four stages.

1. Secret distribution and commitment: Using the paradigm of secure multiparty computation, each participant $U_{i}$ broadcasts $\boldsymbol{w}_{i}$ so that any participant $U_{j}$ can compute $f_{i}\left(\ldots, k_{i}, \ldots\right)=k_{i}$ from $\boldsymbol{w}_{i}$ and his secret $x_{j}$. Since the computation is secure, no passive adversary shall get any information about $k_{i}$. Also, $U_{i}$ broadcasts $\boldsymbol{c}_{i}$ that commits to $k_{i}$ so that other participants can verify correctness of $k_{i}$.

2. Sub-key computation and verification: $U_{i}$ computes $k_{j}^{\prime}$ of all other participants $U_{j}, j \neq i$. When $U_{i}$ gets $k_{j}^{\prime}$, he can use $\boldsymbol{u}_{j}$ to check whether $k_{j}^{\prime}$ is correct. 
3. Fault detection: If the above verification is not correct, $U_{i}$ asks $U_{j}$ to reveal information about commitment $\boldsymbol{c}_{i}$ and messages $\boldsymbol{w}_{i}$ so that all participants can determine whether $U_{i}$ is cheating. If $U_{i}$ detects a cheater, he deletes the cheater from his participant set and restarts the protocol.

4. Conference-key computation: When no faults are detected, add all subkeys together to get the conference key.

Actually, each participant $U_{i}$ can use a different method for securely computing $f_{i}$ and committing to $k_{i}$ as long as the methods are known by other participants.

\section{A Concrete Protocol}

The system has public parameters:

- $p$ : a large prime number that is $2 q+1$, where $q$ is a large prime also.

- $H$ : a one-way permutation from $Z_{q}$ to $Z_{q}$.

$-g$ : a generator (primitive root) for the subgroup $H_{q}$ of quadratic residues of $Z_{p}^{*}$.

Each user $U_{i}$ has two parameters:

- Private parameter $x_{i}$ : a number in $Z_{q}^{*}$.

- Public parameter $y_{i}=g^{x_{i}} \bmod p$. Since $q$ is prime, $y_{i}$ is a generator for $H_{q}$.

The protocol starts with that an initiator calls for a conference for a set $U$ of participants. Without loss of generality, let $U=\left\{U_{1}, U_{2}, \ldots, U_{n}\right\}$ be the initial participant set. Each $U_{i}, 1 \leq i \leq n$, knows $U$.

1. Secret distribution and commitment: each participant $U_{i}$ does the following:

(a) Randomly select $R_{i}, K_{i} \in Z_{q}, S_{i} \in Z_{q}^{*}$.

(b) Compute a polynomial $h_{i}(x)$ (over $Z_{q}$ ) of degree $n$ that passes points $\left(j, y_{j}^{R} \bmod p \bmod q\right), 1 \leq j \leq n$, and $\left(0, K_{i}\right)$.

(c) Compute and broadcast

$$
\begin{gathered}
w_{i j}=h_{i}(n+j) \bmod q, 1 \leq j \leq n, \\
\alpha_{i}=g^{R_{i}} \bmod p, \\
\gamma_{i}=g^{S_{i}} \bmod p \\
\delta_{i}=S_{i}^{-1}\left(H\left(K_{i}\right)-\gamma_{i} x_{i}\right) \bmod q .
\end{gathered}
$$

2. Sub-key computation and verification: each participant $U_{i}$ does the following for $j \neq i$ :

(a) On receiving $w_{j l}, 1 \leq l \leq n$, and $\alpha_{j}$, compute polynomial $h_{j}^{\prime}(x)$ (over $Z_{q}$ ) of degree $n$ that passes $\left(n+l, w_{j l}\right), 1 \leq l \leq n$, and $\left(i, \alpha_{j}^{x_{i}} \bmod p \bmod q\right)$.

(b) Let $K_{j}^{\prime}=h_{j}^{\prime}(0) \bmod q$. 
(c) Check whether $\left(\gamma_{j}, \delta_{j}\right)$ is the ElGamal signature of $H\left(K_{j}^{\prime}\right)$ by $U_{j}$, i.e., check whether $g^{H\left(K_{j}^{\prime}\right)} \bmod p=y_{j}^{\gamma_{j}} \gamma_{j}^{\delta_{j}} \bmod p$. If so, broadcast $V_{i j}=$ "success". Otherwise, broadcast $V_{i j}=$ "failure".

3. Fault detection: each participant $U_{i}$ does the following for $j \neq i$ :

(a) On receiving $V_{j i}=$ "failure" for some $U_{j}$ : $U_{j}$ claims that $U_{i}$ itself is faulty.

i. Output $R_{i}, K_{i}, S_{i}$.

(b) On receiving $V_{j m}=$ "failure": $U_{j}$ claims that $U_{m}, m \neq i$, is faulty.

i. Wait for $U_{m}$ 's fault detection messages $R_{m}, K_{m}, S_{m}$.

ii. If $U_{m}$ 's fault detection messages are not received, set $U_{m}$ as a malicious participant.

iii. On receiving $R_{m}, K_{m}, S_{m}$, check whether $w_{m l}, 1 \leq m \leq n, \alpha_{m}, \gamma_{m}$, and $\delta_{m}$ are correct, i.e., check whether $\alpha_{m}=g^{R_{m}} \bmod p$, whether there is an $n$-degree polynomial over $Z_{q}$ passing points $\left(0, K_{m}\right)$, $\left(l, y_{l}^{R_{m}} \bmod p \bmod q\right)$, and $\left(n+l, w_{m l}\right), 1 \leq l \leq n$, and whether $\left(\gamma_{m}, \delta_{m}\right)$ is the ElGamal signature of $U_{m}$ on $H\left(K_{m}\right)$. If so, set $U_{j}$ as a malicious participant. Otherwise, set $U_{m}$ as a malicious participant.

(c) Restart the protocol by deleting malicious participants from his participant set U.

4. Conference-key computation: If no faults are detected in the fault detection stage, each particpant $U_{i}$ computes the conference

$$
K=\left(K_{i_{1}}^{\prime}+K_{i_{2}}^{\prime}+\cdots+K_{i_{m}}^{\prime}\right) \bmod q
$$

where the current participant set is $U^{\prime}=\left\{U_{i_{1}}, U_{i_{2}}, \ldots, U_{i_{m}}\right\}$.

\section{Security Analysis}

We show security of the above protocol in correctness, fault tolerance and withstanding the attack of passive adversaries.

\subsection{Correctness and Fault Tolerance}

For correctness (completeness) of our protocol, we show that if all participants follow the protocol, they compute a common conference key.

Theorem 1 (Correctness). If all participants follow the protocol, they compute a common conference key.

Proof. ¿From the broadcast messages of participant $U_{j}$, participant $U_{i}$ can compute the polynomial $h_{j}(x) \bmod q$ passing points $\left(n+l, w_{j l}\right), 1 \leq l \leq n$, and $\left(i, \alpha_{j}^{x_{i}} \bmod p \bmod q\right) . U_{i}$ then computes $K_{j}=h_{j}(0) \bmod q$. By the verification messages $\gamma_{j}$ and $\delta_{j}, U_{i}$ can check whether $K_{j}$ is correct. Since for fixed $\gamma_{j}$ and $\delta_{j}$ the signed text $K_{j} \in Z_{q}$ is unique, all participants compute the same $K_{j}$. Thus, they compute the same conference key $K=\left(K_{1}+K_{2}+\cdots+K_{n}\right) \bmod q$.

For fault-tolerance (robustness), we show two things: 
1. Any malicious participant $U_{i}$ who tries to cheat honest participants to accept different $K_{i}$ will be excluded from the participant sets of all honest participants.

2. An honest participant will not be excluded from the participant set of any other honest participant.

Note that it does not matter that a malicious $U_{i}$ causes other malicious participants to compute different $K_{i}$.

Lemma 1. Any malicious participant $U_{i}$ who tries to cheat honest participants to accept different $K_{i}$ shall be excluded from the participant sets of all honest participants.

Proof. Malicious participants can deviate from the protocol in two ways. First, a malicious participant $U_{i}$ sends "wrong" $w_{i l}, 1 \leq l \leq n, \alpha_{i}, \gamma_{i}$ and $\delta_{i}$ so that two honest participants $U_{j}$ and $U_{m}$ compute different $K_{i}$. In this case, one of them, say $U_{j}$, shall send $V_{j i}=$ "failure" since $\gamma_{i}$ and $\delta_{i}$ can not be the ElGamal signature of two different $K_{i}$ 's. Then, $U_{i}$ has to broadcast $R_{i}, K_{i}$ and $S_{i}$ for verification. Every honest participant verifies whether $\alpha_{i}=g^{R_{i}} \bmod p,\left(\gamma_{i}, \delta_{i}\right)$ is the signature of $H\left(K_{i}\right)$, and the polynomial passing $\left(n+l, w_{i l}\right), 1 \leq l \leq n$ and $\left(0, K_{i}\right)$ also passes points $\left(j, y_{j}^{R_{i}} \bmod p \bmod q\right), 1 \leq j \leq n$. Since the honest $U_{j}$ claims that $K_{i}$ is wrong, for all participants at least one of the above checkings cannot hold. Therefore, all honest participants exclude $U_{i}$ from their participant sets.

Second, $U_{i}$ sends $V_{i j}=$ "failure" of claiming that $U_{j}$ is malicious, while $U_{j}$ is indeed honest. In this case, $U_{j}$ broadcasts $R_{j}, K_{j}$ and $S_{j}$ to prove his honesty. Since $U_{j}$ is honest, all honest participants decide that $U_{i}$ is malicious. Therefore, the malicious $U_{i}$ is excluded by all honest participants.

Lemma 2. No honest participant excludes any other honest participant from his participant set.

Proof. Since an honest participant $U_{i}$ follows the protocol, his broadcast messages make all participants compute the same $K_{i}$. Even some malicious participant $U_{j}$ claims that he is faulty, he can send $R_{i}, K_{i}$ and $S_{i}$ to prove his honesty. Therefore, no honest participant shall exclude $U_{i}$ from his participant set.

By the above two lemmas, we can show that all honest participants compute the same conference key even the majority of the participants are malicious.

Theorem 2 (Robustness). All honest participants have the same participant set and thus they compute same conference key no matter how many participants are malicious.

Proof. By the above two lemmas, each honest participant's participant set consists of two types of participants: honest participants and those participants $U_{i}$, though deviating from the protocol, make all honest participants compute the same $K_{i}$. Therefore, all honest participants compute the same conference key. 


\subsection{Security against Passive Attackers}

A passive attacker (eavesdropper) tries to learn information about the conference key by listening the broadcast channel. We show that an eavesdropper cannot get any information about $K_{i}$ of $U_{i}$. Since each participant chooses his $K_{i}$ independently, we show that the attacker's view of the messages broadcast by $U_{i}$ on the broadcast channel can be simulated without knowing the secrets $x_{i}$ and $K_{i}$.

We need an assumption to show that the simulated transcript is computationally indistinguishable from the real one. This assumption is a little stronger than that about the regular Diffie-Hellman decision problem that is discussed in some papers $[6,21,27]$. The assumption about the regular Diffie-Hellman decision problem is that for any given $y_{1}, y_{2} \in H_{q}-\{1\}$ and $u_{1}, u_{2} \in H_{q}$,

$$
\left(y_{1}, y_{2}, y_{1}^{R} \bmod p, y_{2}^{R} \bmod p\right)
$$

and

$$
\left(y_{1}, y_{2}, u_{1}, u_{2}\right)
$$

are computationally indistinguishable and thus

$$
\left(y_{1}, y_{2}, y_{1}^{R} \bmod p \bmod q, y_{2}^{R} \bmod p \bmod q\right)
$$

and

$$
\left(y_{1}, y_{2}, u_{1} \bmod q, u_{2} \bmod q\right)
$$

are computationally indistinguishable. Note that $y_{1}$ and $y_{2}$ must be quadratic residues of $Z_{p}^{*}$, otherwise one can tell apart the above probability distributions. We note that $u_{1} \bmod q$ and $u_{2} \bmod q$ do not range all over $Z_{q}$. Therefore, we need an assumption about a variation of the Diffie-Hellman decision problem.

Assumption 1 (Variant Diffie-Hellman decision problem). Let $p=2 q+1$ and $H_{q}$ be the quadratic-residue subgroup of $Z_{p}^{*}$. Given any generators $y_{1}, y_{2} \in$ $H_{q}-\{1\}$, the following two random-variable tuples are computationally indistinguishable:

$$
\left(y_{1}, y_{2}, y_{1}^{R} \bmod p \bmod q, y_{2}^{R} \bmod p \bmod q\right)
$$

and

$$
\left(y_{1}, y_{2}, u_{1}, u_{2}\right)
$$

where $R, u_{1}, u_{2} \in Z_{q}$.

The simulator of the adversary's view on broadcast messages of $U_{i}$ does the following:

1. Randomly select $w_{i j}^{\prime} \in Z_{q}, 1 \leq j \leq n, R_{i}^{\prime} \in Z_{q}, S_{i}^{\prime} \in Z_{q}^{*}, \delta_{i}^{\prime} \in Z_{q}$,

2. Output the simulated transcript:

$$
\begin{gathered}
w_{i j}^{\prime}, 1 \leq j \leq n, \\
\alpha_{i}^{\prime}=g^{R_{i}^{\prime}} \bmod p, \\
\gamma_{i}^{\prime}=g^{S_{i}^{\prime}} \bmod p, \\
\delta_{i}^{\prime} .
\end{gathered}
$$


We now show, on random variables $K_{i}, R_{i} \in Z_{q}, S_{i} \in Z_{q}^{*}$, the real view

$$
\left(w_{i 1}, w_{i 2}, \ldots, w_{i n}, \alpha_{i}, \gamma_{i}, \delta_{i}\right)
$$

and, on random variables $w_{i j}^{\prime} \in Z_{q}, 1 \leq j \leq n, R_{i}^{\prime} \in Z_{q}, S_{i}^{\prime} \in Z_{q}^{*}, \delta_{i}^{\prime} \in Z_{q}$, the simulated view

$$
w_{i 1}^{\prime}, w_{i 2}^{\prime}, \ldots, w_{i n}^{\prime}, \alpha_{i}^{\prime}, \gamma_{i}^{\prime}, \delta_{i}^{\prime}
$$

are computationally indistinguishable, where $\alpha_{i}=g^{R_{i}} \bmod p, \gamma_{i}=g^{S_{i}} \bmod p$, $\delta_{i}=S_{i}^{-1}\left(K_{i}-\gamma_{i} x_{i}\right) \bmod q, \alpha_{i}^{\prime}=g^{R_{i}^{\prime}} \bmod p, \gamma_{i}^{\prime}=g^{S_{i}^{\prime}} \bmod q$, and $w_{i j}=h_{i}(n+$ $j), 1 \leq j \leq n$, is described in our protocol. Since for any $\gamma_{0} \in H_{q}-\{1\}$ and $\delta_{0} \in Z_{q}$

$$
\operatorname{Pr}\left[\gamma_{i}=\gamma_{0}, \delta_{i}=\delta_{0}\right]=\operatorname{Pr}\left[\gamma_{i}^{\prime}=\gamma_{0}, \delta_{i}^{\prime}=\delta_{0}\right]=\frac{1}{q(q-1)},
$$

we only have to consider the probability distributions

$$
\left.\operatorname{Pr}\left[\left(w_{i 1}, w_{i 2}, \ldots, w_{i n}, \alpha_{i}\right)\right] \gamma_{i}=\gamma_{0}, \delta_{i}=\delta_{0}\right]
$$

and

$$
\operatorname{Pr}\left[\left(w_{i 1}^{\prime}, w_{i 2}^{\prime}, \ldots, w_{i n}^{\prime}, \alpha_{i}^{\prime}\right) \mid \gamma_{i}^{\prime}=\gamma_{0}, \delta_{i}^{\prime}=\delta_{0}\right] .
$$

For any fixed $\gamma_{0}$ and $\delta_{0}$, the random variable $K_{i}$ is fixed, say $k_{0}$. We have

$$
\begin{aligned}
& \operatorname{Pr}\left[\left(w_{i 1}, w_{i 2}, \ldots, w_{i n}, \alpha_{i}\right) \mid \gamma_{i}=\gamma_{0}, \delta_{i}=\delta_{0}\right] \\
& =\operatorname{Pr}\left[\left(w_{i 1}, w_{i 2}, \ldots, w_{i n}, \alpha_{i}\right) \mid K_{i}=k_{0}\right]
\end{aligned}
$$

and

$$
\begin{aligned}
& \operatorname{Pr}\left[\left(w_{i 1}^{\prime}, w_{i 2}^{\prime}, \ldots, w_{i n}^{\prime}, \alpha_{i}^{\prime}\right) \mid \gamma_{0}, \delta_{0}\right] \\
& =\operatorname{Pr}\left[\left(w_{i 1}^{\prime}, w_{i 2}^{\prime}, \ldots, w_{i n}^{\prime}, \alpha_{i}^{\prime}\right)\right]
\end{aligned}
$$

for some $k_{0} \in Z_{q}$. We show that they are computationally indistinguishable.

Lemma 3. Under Assumption 1, for any fixed $K=k_{0}$, on random variables $R_{i}, R_{i}^{\prime}, w_{i 1}^{\prime}, w_{i 2}^{\prime}, \ldots, w_{i n}^{\prime} \in Z_{q}$,

$$
\left(w_{i 1}, w_{i 2}, \ldots, w_{i n}, \alpha_{i}\right)
$$

and

$$
\left(w_{i 1}^{\prime}, w_{i 2}^{\prime}, \ldots, w_{i n}^{\prime}, \alpha_{i}^{\prime}\right)
$$

are computationally indistinguishable.

Proof. By the assumption

$\left(y_{1}, y_{2}, \ldots, y_{n}, y_{1}^{R_{i}} \bmod p \bmod q, y_{2}^{R_{i}} \bmod p \bmod q, \ldots, y_{n}^{R_{i}} \bmod p \bmod q\right.$,

$$
\left.g^{R_{i}} \bmod p\right)
$$

and

$$
\left(y_{1}, y_{2}, \ldots, y_{n}, u_{1}, u_{2}, \ldots, u_{n}, g^{R_{i}^{\prime}} \bmod p\right)
$$


are computationally indistinguishable, where $R_{i}, R_{i}^{\prime}, u_{j} \in Z_{q}, 1 \leq j \leq n$. Let $K=k_{0}$ be fixed. Let $\bar{h}_{i}$ (over $Z_{q}$ ) be the $n$-degree polynomial passing points $\left(0, k_{0}\right)$ and $\left(j, u_{j}\right), 1 \leq j \leq n$. By applying a polynomial interpolation on them, we have that,

$$
\left(w_{i 1}, w_{i 2}, \ldots, w_{i n}, g^{R_{i}} \bmod p\right)
$$

and

$$
\left(\bar{w}_{i 1}, \bar{w}_{i 2}, \ldots, \bar{w}_{i n}, g^{R_{i}^{\prime}} \bmod p\right)
$$

are computationally indistinguishable, where $\bar{w}_{i j}=\bar{h}_{i}(n+j) \bmod q, 1 \leq j \leq n$. Since for any $\bar{w}_{i j}^{0} \in Z_{q}, 1 \leq j \leq n$, and $\bar{\alpha}_{0} \in H_{q}$,

$$
\operatorname{Pr}\left[\left(\bar{w}_{i 1}, \bar{w}_{i 2}, \ldots, \bar{w}_{i n}, g^{R^{\prime}} \bmod p\right)=\left(\bar{w}_{i 1}^{0}, \bar{w}_{i 2}^{0}, \ldots, \bar{w}_{i n}^{0}, \bar{\alpha}_{0}\right)\right]=\frac{1}{q^{n+1}},
$$

thus $\left(w_{i 1}, w_{i 2}, \ldots, w_{i n}, \alpha_{i}\right)$ and $\left(w_{i 1}^{\prime}, w_{i 2}^{\prime}, \ldots, w_{i n}^{\prime}, \alpha_{i}^{\prime}\right)$ are computationally indistinguishable.

Therefore, the simulator outputs a transcript that is computationally indistinguishable from the real one.

Theorem 3 (Privacy). Under Assumption 1, for any $i, 1 \leq i \leq n$, the real communication transcript of $U_{i}$

$$
\left(w_{i 1}, w_{i 2}, \ldots, w_{i n}, \alpha_{i}, \gamma_{i}, \delta_{i}\right)
$$

and the simulated one

$$
\left(w_{i 1}^{\prime}, w_{i 2}^{\prime}, \ldots, w_{i n}^{\prime}, \alpha_{i}^{\prime}, \gamma_{i}^{\prime}, \delta_{i}^{\prime}\right)
$$

are computationally indistinguishable, where random variables $R_{i}, K_{i} \in Z_{q}, S_{i} \in$ $Z_{q}^{*}$ and $w_{i 1}^{\prime}, w_{i 2}^{\prime}, \ldots, w_{i n}^{\prime} \in Z_{q}, S_{i}^{\prime} \in Z_{q}^{*}, \delta_{i}^{\prime} \in Z_{q}$.

Proof. This is obvious by Lemma 3.

\section{Security against Impersonators}

We have discussed the security of our protocol against eavesdroppers and malicious participants. We have assumed that the broadcast channel is authentic. Therefore, no impersonator (outsider) can succeed in pretending to be a legal participant without being detected. It is easy to enforce "authentication" on the broadcast channel since we can simply require participants to sign their broadcast messages. However, it is cumbersome.

In fact, we can relax the requirement of "authenticated" broadcast channel. In our protocol, we require the participant $U_{i}$ to $\operatorname{sign} H\left(K_{i}\right)$, instead of $K_{i}$. The reason is to prevent impersonation of $U_{i}$. We note that an outsider without knowing $U_{i}$ 's secret $x_{i}$ can sign a random message $m=-\gamma_{i} a b^{-1} \bmod p$ by choosing $\gamma_{i}=g^{a} y_{i}^{b} \bmod p$ and $\delta_{i}=-\gamma_{i} b^{-1} \bmod q$ first for $a \in Z_{q}$ and $b \in$ 
$Z_{q}^{*}$ [20]. If we only require $U_{i}$ to $\operatorname{sign} K_{i}=m$, the impersonator can share $K_{i}$ with other participants even though he cannot compute other participants' $K_{j}$ 's.

We don't have a rigid proof for our protocol's strength against impersonators. Nevertheless, we give some explanation. First, if the impersonator chooses $K_{i}$ first and then signs $H\left(K_{i}\right)$, he has to sign a chosen message $H\left(K_{i}\right)$, which is not known to be possible in the ElGamal signature scheme. Second, if the impersonator chooses $m=H\left(K_{i}\right)$ first, he has to compute $K_{i}=H^{-1}(m)$ in order to share $K_{i}$ with other participants. This occurs with only a negligible probability under $H$ being a one-way permutation. A strong evidence shows that this approach (full-domain-hash-then-sign) is secure against signature forgery, thus impersonators [1].

\section{Conclusion}

Assuming an authenticated broadcast channel, we have presented a conferencekey agreement protocol that is provably secure against passive and active adversaries under the assumption of a variant Diffie-Hellman decision problem. We argue that our protocol is secure against active impersonators if the full-domainhash-then-sign paradigm for ElGamal signature is secure.

Our protocol is round-efficient. It uses only two rounds to compute a common conference key after all malicious participants are detected. Nevertheless, the size of messages that each participant sends is proportional to the number of participants. It is interesting to design a provably secure conference-key agreement protocol with both round- and message-efficiency.

\section{References}

1. M. Bellare, P. Rogaway, "The Exact Security of Digital Signatures, How to Sign with RSA and Rabin", Advances in Cryptology: Proceedings of Eurocrypt '96, Lecture Notes in Computer Science 1070, Springer-Verlag, pp.399-416, 199611

2. M. Ben-Or, S. Goldwasser, A. Wigderson, "Completeness Theorems for NonCryptographic Fault-Tolerant Distributed Computation", In Proceedings of the 20th ACM Symposium on the Theory of Computing, pp.1-10, 1988.

3. S. Berkovits, "How to Broadcast a Secret", Advances in Cryptology: Proceedings of Eurocrypt '91, Lecture Notes in Computer Science 547, Springer-Verlag, pp.535541, 1991. 2

4. R. Blom, "An Optimal Class of Symmetric Key Generation Systems", Advances in Cryptology: Proceedings of Eurocrypt '84, Lecture Notes in Computer Science 196, Springer-Verlag, pp.335-338, 1985. 2

5. C. Blundo, A.D. Santis, A. Herzberg, S. Kutten, U. Vaccaro, M. Yung, "PerfectlySecure Key Distribution for Dynamic Conferences", Advances in Cryptology: Proceedings of Crypto '92, Lecture Notes in Computer Science 740, Springer-Verlag, pp.471-486, 1993. 2

6. D. Boneh, R. Venkatesan, "Hardness of Computing the Most Significant Bits of Secret Keys in Diffie-Hellman and Related Problems", Advances in Cryptology: Proceedings of Crypto '96, Lecture Notes in Computer Science 1109, SpringerVerlag, pp.129-142, 1996. 8 
7. M. Burmester, Y. Desmedt, "A Secure and Efficient Conference Key Distribution System", Advances in Cryptology: Proceedings of Eurocrypt '94, Lecture Notes in Computer Science 950, Springer-Verlag, pp.275-286, 1995. 3

8. R. Canetti, A. Herzberg, "Maintaining Security in the Presence of Transient Faults", Advances in Cryptology: Proceedings of Crypto '94, Lecture Notes in Computer Science 839, Springer-Verlag, pp.425-438, 1994.

9. C.C. Chang, C.H. Lin, "How to Converse Securely in a Conference", In Proceedings of IEEE Security Technology, 30th Annual 1996 International Carnahan Conference, pp.42-45, 1996. 2

10. C.C. Chang, T.C. Wu, C.P. Chen, "The Design of a Conference Key Distribution System", Advances in Cryptology: Proceedings of Auscrypt '92, Lecture Notes in Computer Science 718, Springer-Verlag, pp.459-466, 1992. 2

11. W. Diffie, M. Hellman, "New Directions in Cryptography", IEEE Transaction of Information Theory, Vol. IT-22, pp.644-654, 1976.

12. M. Fitzi, M. Hirt, U. Maurer, "Trading Correctness for Privacy in Unconditional Multi-Party Compution", Advances in Cryptology: Proceedings of Crypto '98, Lecture Notes in Computer Science 1462, Springer-Verlag, pp.121-136, 1998.

13. T. Hwang, J.L. Chen, "Identity-Based Conference Key Broadcast Systems", IEE Computers and Digital Techniques, Vol. 141, No. 1, pp.57-60, 1994.

14. I. Ingemarsson, D.T. Tang, C.K. Wong, "A Conference Key Distribution System", IEEE Transactions on Information Theory, Vol. IT-28, No. 5, pp.714-720, 1982. 2

15. B. Klein, M. Otten, T. Beth, "Conference Key Distribution Protocols in Distributed Systems", In Proceedings of Codes and Ciphers-Cryptography and Coding IV, IMA, pp.225-242, 1995. 2

16. K. Koyama, "Secure Conference Key Distribution Schemes for Conspiracy Attack", Advances in Cryptology: Proceedings of Eurocrypt '92, Lecture Notes in Computer Science 658, Springer-Verlag, pp.449-453, 1993. 2

17. K. Koyama, K. Ohta, "Identity-Based Conference Key Distribution Systems", Advances in Cryptology: Proceedings of Crypto '87, Lecture Notes in Computer Science 293, Springer-Verlag, pp.175-184, 1988. 2

18. K. Koyama, K. Ohta, "Security of Improved Identity-Based Conference Key Distributioin Systems", Advances in Cryptology: Proceedings of Eurocrypt '88, Lecture Notes in Computer Science 330, Springer-Verlag, pp.11-19, 1988.

19. T. Matsumoto, H. Imai, "On the Key Predistribution System: A Practical Solution to the Key Distribution Problem", Advances in Cryptology: Proceedings of Crypto '87, Lecture Notes in Computer Science 293, Springer-Verlag, pp.185-193, 1988. 2

20. C.J. Mitchell, F. Piper, P. Wild, "Digital Signature", In Contempary Cryptography, The Science of Information Integrity, pp.325-378, IEEE Press, 1992. 11

21. M. Naor, O. Reingold, "Number-theoretic Constructions of Efficient Pseudorandom Functions", In Proceedings of the 38th IEEE Symposium on Foundations of Computer Science, 1997. 8

22. R. Ostrovsky, M. Yung, "How to Withstand Mobile Virus Attacks", In Proceedings of ACM Symposium on Principles of Distributed Computing, pp.51-61, 1991.

23. T. Rabin, M. Ben-Or, "Verifiable Secret Sharing and Multiparty Protocols with Honest Majority", Proceedings of the 26th ACM Symposium on the Theory of Computing, pp73-85, 1989.

24. R.A. Rueppel, P.C. Van Oorschot, "Modern Key Agreement Techniques", Computer Communications, 1994.

25. A.Shamir, "How to share a secret", Communications of the ACM, Vol. 22, pp.612613, 1979. 
26. A. Shimbo, S. I. Kawamura, "Cryptanalysis of Several Conference Key Distribution Schemes", Advances in Cryptology: Proceedings of Asiacrypt '91, Lecture Notes in Computer Science 739, Springer-Verlag, pp.265-276, 1993.

27. V. Shoup, "Lower Bounds for Discrete Logarithms and Related Problems", Advances in Cryptology: Proceedings of Eurocrypt '97, Lecture Notes in Computer Science 1233, Springer-Verlag, pp.256-266, 1997.8

28. D.G. Steer, L. Strawczynski, W. Diffie, M. Wiener, "A Secure Audio Teleconference System", Advances in Cryptology: Proceedings of Crypto '88, Lecture Notes in Computer Science 409, Springer-Verlag, pp.520-528, 1990. 2

29. T.C. Wu, "Conference Key Distribution System with User Anonymity Based on Algebraic Approach", Proceedings of IEE Computers and Digital Techniques, Vol. 144, No 2, pp.145-148, 1997. 2

30. Y. Yacobi, "Attack on the Koyama-Ohta Identity Based Key Distribution Scheme", Advances in Cryptology: Proceedings of Crypto '87, Lecture Notes in Computer Science 293, Springer-Verlag, pp429-433, 1988. 